\title{
On Some Controllability Conditions for Chaotic Dynamics Control
}

\author{
GUANRONG CHEN† \\ Department of Electrical and Computer Engineering. University of Houston, Houston, TX 77204, USA
}

\begin{abstract}
In this paper, we present some conventional feedback controller design principles for chaos control, with mathematical controllability conditions derived via the Lyapunov function methods. The chaotic Chua's circuit and Duffing oscillator are used as examples to illustrate the fundamental concepts and basic methodology employed by this unified Lyapunov approach, in both linear and non-linear controllers design, for the control of chaotic dynamics. (C) 1997 Elsevier Science Ltd
\end{abstract}

\section{INTRODUCTION}

Conventional feedback controllers are usually designed for non-chaotic systems. In particular, linear feedback controllers are often designed for linear systems. Adaptive feedback controllers are more sophisticated but also more powerful in controlling linear. non-linear and even uncertain systems. That a feedback system is resistant to chaotic signals is perhaps a statement that requires no justification to a dynamics analyst, but deriving a conventional control algorithm ensure the chaotic system trajectory to stay at its unstable periodic orbits (including equilibria and limit cycles) is non-traditional and is not trivial at all. Since conventional feedback control designers generally had no intention of dealing with the extreme sensitivity of chaotic system trajectories to their initial conditions $[2,13,14,16$. 24], it was reasonable to be sceptical about the possibility of applying conventional feedback controls to chaotic systems. However, recent progress has convinced that this is not only possible but, as a matter of fact, has turned out to be successful $[1,4,18]$.

Having observed that some applications of conventional feedback control techniques in chaos control and dynamics monitoring have not had enough theoretical backing to support their success, in this paper we discuss some mathematical controllability conditions for chaotic dynamics control that employ conventional feedback control methods, with the hope that this might stimulate further endeavor on mathematical analysis of chaos control.

Briefly, one basic reason in supporting such success seems to be that chaotic systems, although non-linear, complex and sensitive with many strange behaviors, are deterministic by their very nature. Hence, they obey many deterministic rules that can be utilized in designing controllers for different purposes. Of course, nowadays even chaos itself is still a 'mystery', the role that chaos plays and the role that feedback control plays in the task of controlling chaos have to be further understood and explored. The earlier attempts for using conventional feedback controllers to tackle chaos were motivated by many challenges, and were due to the fact that feedback controllers can be easily realized by circuitry, can perform

†Supported by the US Army research Office under Grant DAAM04-94-G-0227. 
the jobs automatically after being designed and implemented, can stabilize the overall controlled system efficiently and usually have significant physical meanings that make them very useful in real-life applications. Equipped with rigorous mathematical theories, feedback control techniques are deemed to have great potential in chaos control and anti-control, and dynamics monitoring such as bifurcation control and chaos synchronization.

\section{FEFDBACK CONTROL OF CAAOTIC SYSTEMS}

A general approach to controlling a non-linear, complex and perhaps chaotic dynamical system can be formulated as the following control problem: consider a control system

$$
\left\{\begin{array}{l}
\dot{\mathbf{x}}(t)=\mathbf{f}(\mathbf{x}, \mathbf{u}, t) \\
\mathbf{y}(t)=\mathbf{h}(\mathbf{x}, \mathbf{u}, t) .
\end{array}\right.
$$

where $\mathbf{x}(t)$ is the system state vector, $\mathbf{y}(t)$ the output vector and $\mathbf{u}(t)$ the control input vector. Here and throughout the paper, in a general discussion we simply assume all the necessary conditions on the vector-valued functions $\mathbf{f}$ and $\mathbf{h}$ such that the system is well-posed and has a unique solution within a certain region of interest in the state-space for each given initial value $x_{1}=\mathbf{x}\left(t_{1}\right)$ for $t \geq t_{1} \geq 0$. For a given reference signal $\mathbf{r}(t)$. which can be either a constant (set-point) or a function (target trajectory). the problem is to design a controller in the state-feedback form

$$
\mathbf{u}(t)=\mathbf{g}(\mathbf{x}, t)
$$

Or. sometimes, in the output-feedback form

$$
\mathbf{u}(t)=\mathbf{g}(\mathbf{y}, t) .
$$

wherc $\mathbf{g}$ is a linear or non-linear vector-valued function, such that the trajectory of the controlled system

$$
\left\{\begin{array}{l}
\dot{\mathbf{x}}(l)=\mathbf{f}(\mathbf{x}, \mathbf{g}(\mathbf{x}, l), \ell) \\
\mathbf{y}(l)=-\mathbf{h}(\mathbf{x}, \mathbf{g}(\mathbf{x}, \ell)
\end{array}\right.
$$

is driven by the feedback controller $\mathbf{g}(\mathbf{x} . t)$ to achieve a desired goal of tracking:

$$
\lim _{l}\|\mathbf{y}(t)-\mathbf{r}(t)\|=11
$$

where the terminal time. $\Gamma \leq x$, is predesired according to an application in mind, and $\| \cdot i t$ is the standard Euclidean norm of a vector.

Since the second equation in system (1) is merely a mapping. which will not be the main tocus of our discussion below. we simply ignore it by letting $\mathbf{y}=\mathbf{x}$ without loss of any generality.

It is important to note that in the classical control theory, the target for tracking is usually a constant vector in the state space, which is generally not a state of the given system, and the terminal time $T$ is usually finite (e.g. the original concept of 'controllability' is defined using a fixed $T<x$. at lcast for linear systems [13] and for affine-non-linear systems [24]). However, in chaos control, the target for tracking is usually an unstable periodic orbit (or an unstable equilibrium point) of the given system and the terminal time is $T=x$ to be meaningful and practical for the reason that hasic non-linear dynamics such as chaos are asymptotic behaviors.

it is also important to point out that in a feedback controller's design, particularly in 
finding a non-linear controller for a given non-linear system, we must bear in mind that the controller should be (much) simpler than the given system. For instance, if one would like to determine a non-linear controller, say $\mathbf{u}_{k}$ in the discrete-time setting, to guide the state vector $\mathbf{x}_{k}$ of a given non-linear control system in the form

$$
\mathbf{x}_{k+1}=\mathbf{f}_{k}\left(\mathbf{x}_{k}\right)+\mathbf{u}_{k}
$$

to a target trajectory that satisfies a prescribed dynamics $\mathbf{x}_{k+1}=\phi_{k}\left(\mathbf{x}_{k}\right)$, then mathematically it is very easy to use

$$
\mathbf{u}_{k}=\phi_{k}\left(\mathbf{x}_{k}\right)-\mathbf{f}_{k}\left(\mathbf{x}_{k}\right)
$$

which will bring the original system state $\mathbf{x}_{k}$ to the target trajectory in just one step! The problem with this 'design' is that the controller is even more complicated than the given system, and hence has no practical value: it uses the given system to control the given system! It is hard to imagine that one can accept a controller for a machine (such as an aeroplane or a car) that is even bigger than the machine itself. Hence, a successfully designed feedback controller should be as simple as possible: if a linear controller can be found to do the job, use a linear controller; otherwise, try to find a simple non-linear (such as quadratic) controller. Whether or not can one find a simple, easily implementable, low-cost and effective controller for a given task is really an art.

Let us return to the central theme of feedback control for chaos. We first outline a basic idea for the general goal of chaos control that was initially formulated in $[3,5,6]$. Let the target (unstable) periodic orbit of the non-linear dynamical system

$$
\dot{\mathbf{x}}=\mathbf{f}(\mathbf{x}, \mathbf{g}(\mathbf{x}, t), t)
$$

be $\tilde{\mathbf{x}}$, which is itself a solution of the given (uncontrolled) system, that is, it satisfies

$$
\tilde{\mathbf{x}}=\mathbf{f}(\tilde{\mathbf{x}}, 0, t) .
$$

A subtraction of equation (3) from equation (2) now gives

$$
\dot{\mathbf{X}}=\mathbf{F}(\mathbf{X}, t) \text {, }
$$

where

$$
\mathbf{X}:=\mathbf{x}-\tilde{\mathbf{x}}
$$

and

$$
\mathbf{F}(\mathbf{X}, t):=\mathbf{f}(\mathbf{x}, \mathbf{g}(\mathbf{x}, t), t)-\mathbf{f}(\tilde{\mathbf{x}}, 0, t)
$$

Thus, our design is to determine the controller $\mathbf{u}(t)=\mathbf{g}(\mathbf{x}, t)$ such that

$$
\lim _{t \rightarrow \infty}\|\mathbf{X}(t)\|=0
$$

which implies that the goal of tracking control will be achieved:

$$
\lim _{t \rightarrow \infty}\|\mathbf{x}(t)-\tilde{\mathbf{x}}(t)\|=0
$$

It is then clear from equations (4) and (5) that if zero is an equilibrium point of the non-linear system (4), then the original controllability problem has been converted to the 
asymptotic stability problem for this equilibrium. Hence, the Lyapunov second method may be applied or modified to obtain rigorous mathematical techniques for the controller's design. This is further discussed in the rest of this paper.

\subsection{Linear feedhack controllers for non-linear chaotic systems}

It is possible to use a linear feedback controller to control a non-linear chaotic dynamical system in a very rigorous manner.

Take the chaotic Chua's circuit as an example. Chua's circuit is a simple, yet very interesting, electronics system that displays rich and typical bifurcation and chaotic phenomena such as double scroll, dual double scroll and double hook $[20,22,12,27,11]$. In the study of controlling the circuit, we first reformulate its equation in the following form:

$$
\left\{\begin{array}{l}
\dot{x}=p|\cdots+y-f(x)| \\
\dot{y}=x-y+z \\
\dot{z}=-g y
\end{array}\right.
$$

where $p>0, q>0$ and $f(x)$ is a non-linear function represented by

$$
f(x)=m_{0} x+\frac{1}{2}\left(m_{1}-m_{0}\right)(|x+1|-|x-1|) .
$$

where $m_{1}<0$ and $m_{1}<0$.

It is known that with $p=9, q=142 / 7, m_{0}=-5 / 7$ and $m_{1}=-8 / 7$, the circuit displays a double scroll (strange attractor) and an unstable saddle-type periodic orbit, which is a large saddle-type periodic orbit outside the non-periodic attractor and is due to the eventual passivity of the transistors [27].

The following controllability result, using only a lincar feedback controller to guide the chaotic trajectory of the strange attractor to the aforementioned unstable periodic orbit. has been established in [6] hy using the second Lyapunov method:

Theorem $A$. Let $(\bar{x}, \bar{y}, \bar{z})$ be the unstable periodic orbit of Chua's circuit equation (7). Then, the chaotic trajectory $(x, y, z)$ of the circuit can be driven to reach this periodic orbit by a simple linear feedback control of the form

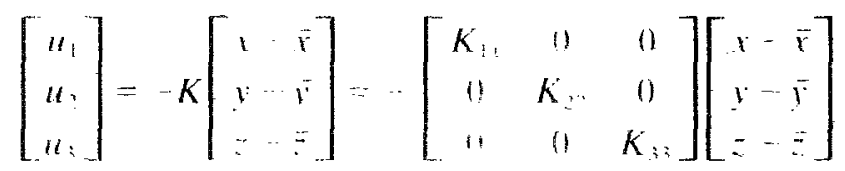

with

$$
K_{1}=-p m_{1}, \quad k_{2}=0 \text { and } K_{3} \geq 0 .
$$

where the control can be applied to the trajectory at any time.

The basic idea for a mathematical proof of this controllability result is described as follows. First, we observe that the controlled circuit is

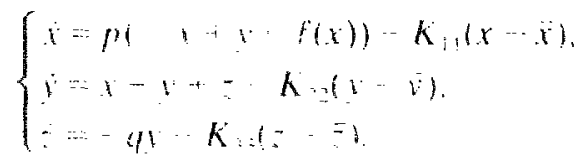

Since the unstable periodic orbit $(\bar{x}, \bar{y}, \bar{z})$ is itself a solution of the circuit. we have

$$
\left\{\begin{array}{l}
\dot{r}=p(\cdots x+y-f(\bar{x})) \\
\dot{y}=-i \cdots= \\
\vdots=-q y
\end{array}\right.
$$


so that a subtraction of equation (10) from equation (9), with the notation

$$
X=x-\bar{x}, \quad Y=y-\bar{y} \quad \text { and } \quad Z=z-\bar{z},
$$

yields

$$
\left\{\begin{array}{l}
\dot{X}=p(-X+Y-\tilde{f}(x, \bar{x}))-K_{11} X \\
\dot{Y}=X-Y+Z-K_{22} Y \\
\dot{Z}=-q Y-K_{33} Z
\end{array}\right.
$$

where

$$
\tilde{f}(x, \bar{x})= \begin{cases}m_{0}(x-\bar{x}) & x \geq 1, \bar{x} \geq 1 \\ m_{0} x-m_{1} \bar{x}+m_{1}-m_{0} & x \geq 1,-1 \leq \bar{x} \leq 1 \\ m_{0}(x-\bar{x})+2\left(m_{1}-m_{0}\right) & x \geq 1, \bar{x} \leq-1 \\ m_{1} x-m_{0} \bar{x}-m_{1}+m_{0} & -1 \leq x \leq 1, \bar{x} \geq 1 \\ m_{1}(x-\bar{x}) & -1 \leq x \leq 1,-1 \leq \bar{x} \leq 1 \\ m_{1} x-m_{0} \bar{x}+m_{1}-m_{0} & -1 \leq x \leq 1, \bar{x} \leq-1 \\ m_{0}(x-\bar{x})-2\left(m_{1}-m_{0}\right) & x \leq-1, \bar{x} \geq 1 \\ m_{0} x-m_{1} \bar{x}-m_{1}+m_{0} & x \leq-1,-1 \leq \bar{x} \leq 1 \\ m_{0}(x-\bar{x}) & x \leq-1, \bar{x} \leq-1\end{cases}
$$

in which $m_{1}<m_{0}<0$.

Recall the general idea discussed in the last section that we want to design the controller by determining the tree constant control gains $\left\{K_{11}, K_{22}, K_{33}\right\}$ such that the zero equilibrium point of the tracking error system equation (11) is asymptotically stable. Observe that this tracking error system is non-autonomous. However, its time-varying and non-linear term is only in $\tilde{f}(x, \bar{x})$ which is connected to the control term $K_{11} X$. Hence, if we can design a control gain $K_{11}$ such that the gain uniformly dominates $\tilde{f}$ with respect to $t \in[0, \infty)$, then the controlled circuit equation (11) will behave like an autonomous system. Based on this observation, we define a simple Lyapunov function for system (11) by

$$
V(X, Y, Z)=\frac{q}{2} X^{2}+\frac{p q}{2} Y^{2}+\frac{p}{2} Z^{2}
$$

It is clear that $V(0,0,0)=0$ and $V(X, Y, Z)>0$ for all $X, Y, Z$, not simultaneously zero. On the other hand, since $p, q>0$ and $K_{22}, K_{33} \geq 0$, we have

$$
\begin{aligned}
\dot{V} & =q X \dot{X}+p q Y \dot{Y}+p Z \dot{Z} \\
& =q X\left(-p X+p Y-p \tilde{f}(x, \bar{x})-K_{11} X\right) \\
& =p q Y\left(X-Y+Z-K_{22} Y\right)+p Z\left(-q Y-K_{33} Z\right) \\
& =-p\left[q(X-Y)^{2}+q K_{22} Y^{2}+K_{33} Z^{2}\right]-q\left(p X \tilde{f}(x, \bar{x})+K_{11} X^{2}\right) \\
& \leq-p\left[q(X-Y)^{2}+q K_{22} Y^{2}+K_{33} Z^{2}\right] \\
& \leq 0
\end{aligned}
$$

for all $X, Y$ and $Z$, if

$$
p X \tilde{f}(x, \bar{x})+K_{11} X^{2} \geq 0
$$


for all $x$ and $\bar{x}$, uniformly with respect to time. It is clear from both $V$ and $\dot{V}$ [see equation (12) $]$ that their uniform boundedness can be easily made rigorous by introducing three class- $K$ functions $[19,26]$.

To find the conditions under which equation (13) is true, by careful examination of the nine possible cases for the function $f(x, \tilde{x})$ shown above, we obtain the final common condition

$$
K_{11} \geq \max \left\{-p m_{0},-p m_{1}\right\}=-p m_{1},
$$

in which we should note that $m_{1}<m_{0}<0$, as indicated above. This condition guarantees the inequality equation (13). Hence, if the conditions stated in Theorem A are satisfied, then the zero equilibrium point of the controlled circuit equation (11) is globally asymptotically stable, so that

$$
|X| \rightarrow 0, \quad|Y| \rightarrow 0, \quad|Z| \rightarrow 0 \quad \text { as } t \rightarrow \infty,
$$

simultaneously. That is, starting the feedback control at any time on the chaotic trajectory, we have

$$
\lim _{t \rightarrow \infty}|x(t)-\bar{x}(t)|=0, \quad \lim _{t \rightarrow \infty}|y(t)-\bar{y}(t)|=0, \quad \lim _{t \rightarrow \infty}|z(t)-\bar{z}(t)|=0 .
$$

We remark that this controllability result provides only some sufficient conditions, whereas the condition on $K_{11}$ may not be necessary from our experience. Indeed, we have found that the control parameters in $K$ can be further reduced. For example, we have experienced that $\left\{K_{11}=0, K_{22}=2, K_{33}=0\right\}$ is perhaps the simplest yet best choice for the feedback control gain matrix $K$ according to our computer simulations [7].

We also remark that perhaps due to the extreme sensitivity of chaos to numerical roundoff errors [23], sometimes in a certain range of the above sufficient conditions the control effect is not so satisfactory. This has been experienced to be true not only for Chua's circuit but also for other chaotic systems.

\subsection{Non-linear feedback controllers for non-linear chaotic systems}

It is not always possible to use a linear controller to drive a non-linear, particularly chaotic, dynamical system [17]; so non-linear feedback controllers are often necessary.

Let us now consider the well-known Duffing oscillator

$$
\left\{\begin{array}{l}
\dot{x}=y \\
\dot{y}=-p_{2} x-x^{3}-p_{1} y+q \cos (\omega t)
\end{array}\right.
$$

where $p_{1}, p_{2}, q$ and $\omega$ are systems parameters. It is well known that with the parameters set at $p_{1}=0.4, p_{2}=-1.1, q=2.1$ (or $q=1.8$ ) and $\omega=1.8$, the Duffing oscillator has a chaotic response [5].

For this system, we are interested in controlling its chaotic trajectory to one of the inherent unstable periodic orbits (limit cycles) of the system by designing a conventional feedback controller.

Let $(\bar{x}, \bar{y})=(\bar{x}(t), \bar{y}(t))$ be the target trajectory: one of its unstable periodic orbits. We want to control the system trajectory, such that

$$
\lim _{t \rightarrow T}|x(t)-\bar{x}(t)|=0 \quad \text { and } \quad \lim _{t \rightarrow T}|y(t)-\bar{y}(t)|=0
$$

for a $T \leq x$. For this purpose, consider the conventional non-linear feedback controller of the general form

$$
u(t)=h(t ; x, \vec{x})
$$


where $h$ is a non-linear function to be determined. By adding this controller to the second equation of the given system, we obtain the following controlled Duffing system:

$$
\left\{\begin{array}{l}
\dot{x}=y \\
\dot{y}=-p_{2} x-x^{3}-p_{1} y+q \cos (\omega t)+h(t ; x, \bar{x}) .
\end{array}\right.
$$

Since the periodic orbit $(\bar{x}, \bar{y})$ is itself a solution of the original system, subtracting equation (15), with $(x, y)$ being replaced by $(\bar{x}, \bar{y})$ therein, from equation $(17)$, and using the notation

we arrive at

$$
X=x-\bar{x} \quad \text { and } \quad Y=y-\bar{y},
$$

$$
\left\{\begin{array}{l}
\dot{X}=Y-p_{1} X \\
\dot{Y}=-p_{2} X-\left(x^{3}-\bar{x}^{3}\right)+h(x)
\end{array}\right.
$$

The following sufficient condition has been established in [5] using the idea of the Lyapunov second method with a specially constructed Lyapunov function.

Theorem $B$. For any (unstable) periodic solution $\bar{x}$ of the Duffing oscillator, let the feedback controller be

$$
u(t)=-K[x(t)-\bar{x}(t)]+h(t ; x, \bar{x}),
$$

where $K \geq p_{2}$ and $h(t ; x, \bar{x})$ satisfies

(i) $h(t ; x, \bar{x})-3 x \bar{x}(x-\bar{x}) \in L_{1}\left(\left[t_{1}, \infty\right) \times S\right)$ and

(ii) $u \in L_{1}\left(\left[t_{1}, x\right)\right)$,

in which $S$ is the domain on which the system is defined. Then, starting from anywhere of the system trajectory at any time $t=t_{1} \geq t_{10}$, the controlled trajectory of the Duffing system converges to the desired periodic orbit, $\bar{x}$, in the sense that

$$
\lim _{t_{1} \leq t \rightarrow \infty}|x-\bar{x}|=0 \quad \text { and } \quad \lim _{t_{1} \leq t \rightarrow \infty}|\dot{x}=\dot{x}|=0 .
$$

A non-linear controller can be designed relatively easily. For example, if we pick [15]

$$
h(t ; x, \bar{x})=K(x-\bar{x})+3 \bar{x}^{2}(x-\bar{x})+3 \bar{x}(x-\bar{x})^{2},
$$

then, using this particular non-linear controller, the tracking error system equation (18) reduces to

$$
\left\{\begin{array}{l}
\dot{X}=Y-p_{1} X, \\
\dot{Y}=-\left(K+p_{2}\right) X-X^{3} .
\end{array}\right.
$$

Observe that this is an autonomous system, and so we can easily find the following Lyapunov function

$$
V(X, Y)=\frac{K+p_{2}}{2} X^{2}+\frac{1}{4} X^{4}+\frac{1}{2} Y^{2}
$$

which satisfies $\dot{V} \leq 0$, where equality holds if and only if both $X \equiv 0$ and $Y \equiv 0$. This means that the zero equilibrium point of the controlled Duffing system equation (20) is asymptotically stable, so that $X \rightarrow 0$ and $Y \rightarrow 0$ as $t \rightarrow \infty$, or the goal

$$
|x-\bar{x}| \rightarrow 0 \quad \text { and } \quad|\dot{x}-\dot{\bar{x}}| \rightarrow 0 \quad(t \rightarrow \infty)
$$

is achieved.

It has been experienced that, in general, a linear feedback controller is much harder 
(often, impossible) to design for the same purpose, where we generally do not have a routine procedure to follow. However, we would like to mention that for the Duffing system. a successful design of a linear feedback controller is still possible which, indeed, has been designed in [5], where the target orbit can even be multi-periodic. The strategy used in [5] is to apply the Lyapunov first method to start the design, so as to allocate some approximate ranges for the controller's gains, and then fine-tune the control gains to achieve the goal. This linear feedback controller has also been verified by other researchers [25], using the same Lyapunov function argument but from a different approach.

\section{SOME GENERAL CONTROLLABILITY CONDITIONS}

As mentioned above, a linear feedback controller alone is not always sufficient for the control of a non-linear chaotic system. It is hence important to find some new criteria for the design of (simple) non-linear feedback controllers used for guiding trajectories of general chaotic dynamical systems to target their unstable periodic orbits. A recent result derived in $[8]$ is described in the following.

Consider a non-linear and non-autonomous dynamical system

$$
\dot{\mathbf{x}}=\mathbf{f}(\mathbf{x}, t)
$$

which is assumed to possess a periodic orbit $\tilde{\mathbf{x}}$ of period $T: \tilde{\mathbf{x}}(t+T)=\tilde{\mathbf{x}}(t)$ for all $0 \leq t<x$. We want to design a feedback controller of the form

$$
\mathbf{u}(l)=K(\mathbf{x}-\tilde{\mathbf{x}})+\mathbf{g}(\mathbf{x}-\tilde{\mathbf{x}}, l)
$$

where $K$ is a constant matrix and $\mathbf{g}$ is a non-linear vector-valued function, and then add it to the original system to obtain

$$
\dot{\mathbf{x}}=\mathbf{f}(\mathbf{x}, t)+\mathbf{u}=\mathbf{f}(\mathbf{x}, t)+K(\mathbf{x}-\tilde{\mathbf{x}})+\mathbf{g}(\mathbf{x}-\tilde{\mathbf{x}}, t) .
$$

The controller is required to be able to drive the trajectory of the controlled system equation (23) to approach the target periodic orbit $\tilde{\mathbf{x}}$. in the sense that

$$
\lim _{i \rightarrow \infty}\|\tilde{\mathbf{x}}(t) \cdots \tilde{\mathbf{x}}(t)\|=0
$$

where, again, $\|\cdot\|$ is the Euclidean norm.

Since the target periodic orbit $\tilde{\mathbf{x}}$ is itself a solution of the original system, it satisfies

$$
\dot{\tilde{\mathbf{x}}}=\mathbf{f}(\tilde{\mathbf{x}}, t) \text {. }
$$

and since the feedback controlled system with the controller equation (22) is given by

$$
\dot{\mathbf{x}}-\mathbf{f}(\mathbf{x}, t)+\dot{K}(\mathbf{x}-\tilde{\mathbf{x}})+\mathbf{g}(\mathbf{x}-\tilde{\mathbf{x}}, t)
$$

a subtraction of equation (25) from equation (26) gives

$$
\dot{\mathbf{X}}=\mathbf{F}(\mathbf{X}, t)+K \mathbf{X}+\mathbf{g}(\mathbf{X}, t)
$$

where

$$
\mathbf{X}=\mathbf{x}-\tilde{\mathbf{x}} \text { and } \mathbf{F}(\mathbf{X}, t)=\mathbf{f}(\mathbf{x}, t)-\mathbf{f}(\tilde{\mathbf{x}}, t)
$$

It is clear that $\mathbf{F}(0, t)=0$ for all $t \in[0, \infty)$.

Next. we Taylor-expand the right-hand side of the controlled system (27) at $\mathbf{X}=0$ 
(i.e. at $\mathbf{x}=\tilde{\mathbf{x}}$ ) and suppose that the non-linear controller that we will design satisfies $\mathbf{g}(0, t)=0$. Then we obtain

where

$$
\dot{\mathbf{X}}=A(\tilde{\mathbf{x}}, t) \mathbf{X}+\mathbf{h}(\mathbf{X}, K, t),
$$

$$
A(\tilde{\mathbf{x}}, t)=\left.\frac{\partial \mathbf{F}(\mathbf{X}, t)}{\partial \mathbf{X}}\right|_{\mathbf{x}=t}
$$

and $\mathbf{h}(\mathbf{X}, K, t)$ is the rest of the Taylor expansion, which is a function of $t, K$ and $O(\mathbf{X})$.

To this end, our design is to determine both the constant control gain matrix $K$ and the non-linear controller $\mathbf{g}(\mathbf{X}, t)$, based on the linearized model equation (28), such that $\mathbf{X} \rightarrow 0$ (i.e. $\mathbf{x} \rightarrow \tilde{\mathbf{x}}$ ) as $t \rightarrow \infty$, so that when being applied to the original system as shown in equation (26), the goal equation (24) can be achieved.

The following two controllability conditions have been established in [8]:

Theorem $C 1$. Suppose that in system $(28) \mathbf{h}(0, K, t)=0$ and $A(\tilde{\mathbf{x}}, t)=A$ is a constant matrix whose eigenvalues all have negative real parts. Let $P$ be a positive definite and symmetric solution (which is known to exist always) of the Lyapunov equation

$$
P A+A^{\top} P=-I,
$$

where $l$ is the identity matrix, with the maximum eigenvalue $\lambda_{\text {max }}(P)>0$. If

$$
\|\mathbf{h}(\mathbf{X}, K, t)\| \leq c\|\mathbf{X}\|
$$

for a constant $c<1 / 2 \lambda_{\max }(P)$ for all $0 \leq t<\infty$, where $\|\cdot\|$ is the Euclidean norm, then the controller $\mathbf{u}(t)$ defined in equation (22) will drive the trajectory $\mathbf{x}$ of the controlled system equation (26) to the target orbit $\tilde{\mathbf{x}}$ as $t \rightarrow \infty$.

To state the next theorem, we first recall a concept from linear algebra. For system equation (28), there is always a $T$-periodic non-singular matrix $M(\tilde{\mathbf{x}}, t)$ and a constant matrix $Q$ such that the fundamental solution matrix associated with the matrix $A(\tilde{\mathbf{x}}, t)$ is given by

$$
\Phi(\tilde{\mathbf{x}}, t)=M(\tilde{\mathbf{x}}, t) \mathrm{e}^{r Q} .
$$

Moreover, the eigenvalues of $\mathrm{e}^{T Q}$ are called the multipliers of the system matrix $A(\tilde{\mathbf{x}}, t)$.

Theorem C2. In system (28), suppose that $\mathbf{h}(0, K, t)=0$, and that $\mathbf{h}(\mathbf{X}, K, t)$ and $\partial \mathbf{h}(\mathbf{X}, K, t) / \partial \mathbf{X}$ are both continuous in a bounded region $\|\mathbf{X}\|<\infty$. Assume moreover that

$$
\lim _{\|\mathbf{X}\| \rightarrow 0} \frac{\|\mathbf{h}(\mathbf{X}, K, t)\|}{\|\mathbf{X}\|}=0,
$$

uniformly with respect to $t \in[0, \infty)$. Then the non-linear controller equation (22) designed under the condition that all the multipliers of the system equation (28) satisfy

$$
\left|\lambda_{i}\right|<1, i=1, \cdots, n, \forall t \in[0, \infty),
$$

will drive the orbit $\mathbf{x}$ of the original controlled system equation (26) to the target orbit $\tilde{\mathbf{x}}$ as $t \rightarrow x$.

\section{CONCLUSIONS}

In this paper, we have presented some conventional feedback controller design principles for chaos control, with mathematical controllability conditions derived from the Lyapunov function methods. The chaotic Chua's circuit and Duffing oscillator were used as examples 
to illustrate the fundamental concepts and basic methodology employed by this unified Lyapunov approach, in the control of chaos by linear and non-linear feedback controls.

Since even the nature of chaos itself is still under intensive investigation, the role that chaos plays and the role that feedback control plays in the task of controlling chaos are not so clear to us at this stage. In particular, how to utilize the very nature of chaos in its control is an open area that calls for more research. Recently, we have started to tackle the problem of directly monitoring the Iyapunov exponents of a dynamical system via conventional feedback control strategies, by applying some rigorous mathematical arguments $[9,10]$. This has led to anti-control of chaos and has also brought in some new ideas and new techniques that employ fundamental characteristics of chaos throughout a control process. It is our hope that control and anti-control of chaos will not only lead to some unique theories that are distinguishable from conventional control concepts, but also lead to some special applications of chaos control that are not conventional.

\section{REFERENCES}

(Chen, ( $i .$, Control and synchronization of chaotic systems (a bibliography). ECE Department. University of Houston, TX-available from ftp: ftp.egr.uh.edu/pub/TeX/chaos.tex (login name: anonymous: password: your inail address).

2. Chen, G., Chen, G. and Hsu, S. H, Linear Stochastic Control Systems. CRC Press, Boca Raton. FL, 1995.

3. Chen, (i. and Dong. X.. On feedhack control of chaotic dynamical svstems. Int. I. Bifurcation and Chaos, 1992. 2. $407-411$.

4. Chen. $G$. and Dong, $X$.. From chaos to order-perspectives and methodologies in controlling nonlinear dynamical systems. Int. J. Bifurcation and Chaos, 1993 3, 1363-1409.

5. Chen, G. atud Dong. X. On feedhack control of chatic continuous-lime systems. IEEE Trans. Circ. Syst. (I), $[693.40,591-60)$.

6. Chen. G. and Dong, X., Controlling Chua's circuit. J. Circ. Syst Comput. 1993, 3, 139-149.

7. Chen. G. and Dong, X.. Ordering chaos of Chua's circuita feedback control approach. Proc. of IEEE Int. Sump. on Circ. Syst. Chicago, IL. May 1993, pp. 2604-2607.

$x$. Chen, $G$, and Hu, Gi., Some controtlability conditions for nonlinear feedback control of chaotic systems. $J$ Berimg Normal Unin. (Naturai Si.i). 1997, 32, 208-212.

i. Chen, G. and Lai, D., Feedback control of Lyapunov exponents for discrete-time dynamical systems. Int. J. Bifurcation and Chaos, 1996, 6, 1341-1349.

10. Chen, G. and Lai, D., Making a dynamical system chaotic: leedback control of Lyapunov exponents for discrete-tıne dynamical systems. IEEE Trans. Circ. Syst. (I). 1997, in press.

11. Chuat. L. O., The genesis of Chua's circuit. AEÜ. Int. J. Elec. Commun., 1992, 46, 187-257.

12. Chua, L. O. Komuro, M. and Matsumoto. T. The double scroll family: I and H. IEEE Trans. Circ. Syst. I986, 33, $10721118^{2}$

13. Chui, C.K. and Chen, (i, Lintear Systems and Optimal Control. Springer, New York, 1989.

14. Chui, C.K. and (hen, G., Signal Processing and Systems Theory: Selected Topics. Springer, New York, 1992.

15. Dong, $X$ and chen, G. Gontrolling chaotic continuous-time systems via feedback. IEEE Proc. Decis. Control Conf., Tueson, AZ, Dec. 1992, pp. 2502-2503.

16. de Figueiredo, R. J. P. and Chen. G., Analysis of Nonlinear Feedhack Control Systems: An Operator Theoretic Approach. Academic Press, New York. 1993.

17. Hu, G., Qu, Z and Chen, G., Stability criterion of a periodic orhit-controllability of chaos. J. Beijing Normal Liniversity (Natural Science), 1995, 31,65-70.

18. Kapitaniak. T., Controlling Chaos. Academic Press, New York, 1996.

19. Khalil, H. K., Nonlinear Systems. Macmillan, New. York, 1995, 2nd edn.

20. Matsumoto, T., A chaotic attractor from Chua's circuit. IEEE Trants. Circ. Syst. 1984, 31. 1055-1058

21. Matsumoto, T., Chaos in electronic circuits. Proc. of IEEE, 1987, 75, 1033-1057.

22. Matsumoto. T., Chua, L. O. and Komuro. M.. The double scroll. IEEE Trans. Cire. Syst., 1985, 32, 798 -817.

23. Mrozek, M., Rigorous numerics of chaotic dynamical systems. In Chaos-The Interplay Between Stochastic and Deterministic Behaviour. eds P. Gabaczewski, M. Wolf and A. Weron. Springer, New York, 1995, pp. 283-296.

3. Nijmeijer. H. and van der Schaft, A. I. Nonlinear Dynamical Control Systems. Springer. New York. 1990.

25. Nijmeijer, H. and Berghuis. H.. On L.yapunov control of the Duffing equation. IEEE Trans. Circ. Syst., 1995. 42, $473-477$.

26. Vidyasagar, M., Nontinear Systems Analysis. Wiley, New York, 1993. 2nd edn.

77. Wu. S.. Chua's circuit family. IEEE Proc., 1987, 75, 1022-1032 